\title{
GENERIC HYDRODYNAMIC INSTABILITY OF CURL EIGENFIELDS
}

\author{
JOHN ETNYRE AND ROBERT GHRIST
}

\begin{abstract}
We prove that for generic geometry, the curl-eigenfield solutions to the steady Euler equations on $\mathbb{R}^{3} / \mathbb{Z}^{3}$ are all hydrodynamically unstable (linear, $L^{2}$ norm). The proof involves a marriage of contact topological methods with the instability criterion of Friedlander-Vishik. An application of contact homology is the crucial step.
\end{abstract}

\section{INTRODUCTION AND STATEMENT OF RESULTS}

The problem of linear hydrodynamic instability for steady Euler flows on three-dimensional domains is classical in nature and foundational in implication. It is universally asserted that in dimension three such flows are almost always unstable, though the precise definition of "almost always" is an issue left undiscussed. The small literature on generic properties of fluid flows [11, 23] focuses on the Navier-Stokes setting and uses external forcing or Dirichlet data as a parameter.

We present a clear formulation of the problem and prove a generic instability theorem for a large class of flows - the curl eigenfields - which form the most fascinating and challenging steady solutions to the Euler equations. The chief difficulty with genericity issues for curl eigenfields is that the "space of all eigenfields" on a typical Riemannian three-manifold is a "discrete" space and is not amenable to perturbations. Our idea in formulating a well-defined genericity statement is to use the geometry of the domain as a parameter.

Main Theorem: For a generic set of $C^{r}$ Riemannian metrics on $T^{3}:=\mathbb{R}^{3} / \mathbb{Z}^{3}$ (for each $2 \leq r<\infty$ ), all of the curl-eigenfield solutions to the Euler equations (with nonzero eigenvalue) are linearly hydrodynamically unstable in energy norm.

Here and throughout the paper, the term generic is interchangeable with the term residual: a subset $A \subset X$ is residual if it is a countable intersection

1991 Mathematics Subject Classification. Primary: 76E09, 37J55; Secondary: 76B99, 53D40.

Key words and phrases. hydrodynamic instability; Beltrami field; Reeb field; contact homology.

JE supported in part by NSF Grant \# DMS - 0203941 .

RG supported in part by NSF Grant \# DMS - 0134408. 
of open dense subsets of $X$. All of the function spaces dealt with in this paper (spaces of vector fields, etc.) are Baire spaces, implying that residual subsets are dense, though not necessarily open. We use the language and notations of differential forms throughout the paper: $d$ denotes the exterior derivative, $\iota$ denotes contraction, $*$ denotes the Hodge-star operator, $\delta:=$ $* d *$ is a codifferential, and $\Delta:=d \delta+\delta d$ is the Laplacian.

Given the recent excellent surveys on the intricacies of the instability problem [12, 14], there is little need to reintroduce the perspectives in detail. In brief, a vector field $u$ is a solution to the steady Euler equations if

$$
(u \cdot \nabla) u=-\nabla p ; \nabla \cdot u=0,
$$

for some real-valued pressure function $p$. Given such a solution, $u$ is said to be linearly stable if, for every sufficiently small divergence-free field $v(0)$, the evolution of $v(t)$ under the linearized Euler equation about $u$,

$$
\frac{\partial v}{\partial t}+(u \cdot \nabla) v+(v \cdot \nabla) u=-\nabla p
$$

is bounded in some predetermined norm. For the remainder of this work, we will, following [12, 13], use the energy $\left(L^{2}\right)$ norm on vector fields. The solution $u$ is said to be linearly unstable if, for some $v(0)$, the solution $v(t)$ has unbounded growth in the chosen norm.

Thanks to an insight of Arnold and the analysis of Friedlander-Vishik [13] (who used the technique developed by Lifshitz-Hameiri [21]), it is now known that the underlying dynamics of the flowlines of the steady solution $u$ can force linear instability. In particular, we rely on the following:

Instability Criterion: [13] The presence of a nondegenerate periodic orbit of hyperbolic (saddle) type in a steady Euler flow induces linear instability in the energy norm.

The outline of the proof of the Main Theorem is as follows: First, we show that for a generic metric, all of the fixed points of all the curl-eigenfields in that metric are nondegenerate. Any nondegenerate fixed point is immediately of saddle type (since the flow is divergence-free) and thus forces instability. This, then, provides a quick proof of generic instability for invariant flow domains such as $S^{2} \times[0,1]$ which are forced to have fixed points on the boundaries.

Most flow domains, however, have vanishing Euler characteristic and thus admit flows without fixed points. The crux of the difficulty is determining when an eigenfield without fixed points possesses a saddle type periodic orbit. We show that in the absence of fixed points, all of the periodic orbits are generically nondegenerate. But how does one determine if periodic orbits exist and are of saddle type? To answer this last, most difficult question, we turn to contact topological methods, including the recently 
announced contact homology of Eliashberg, Givental, and Hofer [7]. For an introduction to contact topological techniques in fluid dynamics, see [8, 15].

We restrict attention in this work to $\mathbb{R}^{3}$ with periodic boundary conditions: $T^{3}$. The sole impediment to applying the proof to arbitrary threedimensional domains is the computation of the contact homology. For domains with boundary which admit a nonvanishing vector field (such as a solid torus), the techniques of [9] should suffice to adapt the proofs to this setting.

The Instability Criterion exists in a slightly more general form: a nonperiodic orbit having a positive Lyapunov exponent is sufficiently expanding to push through the analysis. We have chosen to focus on instances of strict recurrence (fixed points and periodic orbits).

Finally, our results would be much improved by changing the genericity parameter to be the shape of the boundary (the space of embeddings of the boundary into a fixed Euclidean space). That this is possible is asserted in [24] and explored in [17] in the context of eigenfunctions of the Laplace operator. We do not treat this important case in this paper.

\section{GENERIC EIGENFIELDS}

This section presents the basic notation and definitions, then continues with the principal technical lemmas.

2.1. Curl eigenfields. We begin with the class of curl eigenfield solutions to the Euler equations on a compact boundaryless three-manifold $M$ with Riemannian metric $g$ and volume form $\mu$ (assumed to be the volume form derived from $g$ for simplicity — this is not entirely necessary [8]). The vector field $u$ is a curl eigenfield of eigenvalue $\lambda$ iff

$$
\lambda \mu(u, \cdot, \cdot)=d(g(u, \cdot)),
$$

or, in forms notation, if $* d \alpha=\lambda \alpha$, where $\alpha:=\iota_{u} g$ is the dual 1-form to $u$ and $* d$ is the curl operator on 1 -forms. See [3] for the notation used in geometric fluid dynamics on Riemannian manifolds.

A Beltrami field on $M$ is defined to be a volume-preserving vector field $u$ whose curl is parallel to $u$ : in other words, $f \iota_{u} \mu=d\left(\iota_{u} g\right)$ for some map $f: M \rightarrow \mathbb{R}$. It is a well-known fact that $f$ is an integral for the flow of $u$ : its values are fixed along orbits of $u$. Thus, in the case when $f$ is not constant on open sets, the flowlines are restricted to invariant 2-tori almost everywhere. This is a very restrictive assumption, and is in fact not globally possible on most (e.g., hyperbolic) 3-manifolds. For a typical Riemannian manifold (e.g., one without too many symmetries), the moduli space of curl eigenfields is discrete (since the curl squared is essentially the Laplacian 
operator and thus possesses discrete spectrum). The class of Beltrami fields can be significantly larger in the case where the dynamics is integrable.

The Instability Criterion requires some expanding dynamics within the flow, the simplest examples of which are fixed points and periodic orbits which are nondegenerate and of saddle-type. A nondegenerate fixed point is one whose eigenvalues are all nonzero. A nondegenerate periodic orbit for a Hamiltonian flow is defined to be one whose Floquet multipliers (eigenvalues of the linearized return map to a cross-section of the orbit) are not equal to one.

2.2. Reeb fields. Our analysis of curl-eigenfields is strongly rooted in methods derived from contact geometry. For an introduction to contact geometry, see [1, Ch. 8]. In brief, a contact form on a 3-manifold $M$ is a 1-form $\alpha \in \Omega^{1}(M)$ such that $\alpha \wedge d \alpha$ is nowhere vanishing. A contact structure on $M$ is a smooth plane field on $M$ which is (locally) the kernel of a contact 1-form. Two fundamental examples of contact structures are the kernel of $d z+x d y$ on $\mathbb{R}^{3}$ and also the plane field orthogonal to the fibers of the Hopf fibration of the unit $S^{3}$ in Euclidean $\mathbb{R}^{4}$.

To every contact form $\alpha$ is associated a unique vector field, called the Reeb field, which captures the geometry of the 1-form in the directions transverse to the contact structure. The Reeb field of $\alpha$, denoted $X$, is defined implicitly via the two conditions:

$$
d \alpha(X, \cdot)=0 \quad ; \quad \alpha(X)=1 .
$$

The dynamics of the Reeb field, together with the geometry of the contact structure, suffice to reconstruct the contact 1-form.

Contact geometry enters fluid dynamics via the following results [8]. For every nonvanishing curl eigenfield $u$ on $\left(M^{3}, g\right)$ with eigenvalue $\lambda \neq 0$, the dual 1-form $\alpha:=\iota_{u} g$ is a contact 1-form since $\alpha \wedge d \alpha=\lambda \alpha \wedge * \alpha \neq 0$. Furthermore, the Reeb field of $\alpha$ is a rescaling of $u$. Conversely, for each contact form $\alpha$, there is a natural adapted Riemannian metric making the Reeb field an eigenfield of the curl operator in that metric:

$$
g(v, w):=(\alpha(v) \otimes \alpha(w))+d \alpha(v, J w),
$$

where $J$ is any almost-complex structure on $\xi=\operatorname{ker} \alpha$ (a bundle isomorphism $J: \xi \rightarrow \xi$ satisfying $J^{2}=-$ ID) adapted to $d \alpha$.

The more general version of this correspondence theorem can be used to understand the existence and qualitative behavior of steady solutions to the Euler equations [8, 9, 10]. For example, by exploiting the flexibility of contact forms, one can construct steady Euler flows on a 3-d Riemannian ball which possesses periodic flowlines exhibiting all knot and link types [10]. 
Our strategy for the remainder of the paper is first to attack the case of curl-eigenfields with fixed points using simple genericity results. The remaining case concerns (rescalings of) Reeb fields for contact forms. We will use contact-topological methods in this last case. These topological methods also require a certain degree of nondegeneracy, and thus necessitate genericity statements.

2.3. Genericity theorems. The following technical result provides the basis for the instability theorem.

Theorem 2.1. For generic choice of $C^{r}$ metric (for each $2 \leq r<\infty$ ), all of the curl-eigenfields on a compact three-manifold $M$ with non-zero eigenvalue have all fixed points nondegenerate. In addition, if any eigenfield does not possess any fixed points, then all its periodic orbits are nondegenerate.

The proof will be detailed through a series of lemmas. The first two lemmas establish that generically all non-zero eigenvalues are simple and eigenfields are transverse to the zero section. We adapt Uhlenbeck's techniques [24] to our situation with two major modifications: (1) we work with vector fields rather than functions; (2) we use the curl operator rather than the Laplacian. Moreover, to simplify the analysis we consider the dual situation by regarding the curl operator as $\nabla \times:=* d$ on the space of 1-forms instead of on vector fields. Denote the space of Riemannian metrics on $M$ by $\mathcal{G}$ and let

$$
E_{0}:=\left\{(g, \alpha) \in \mathcal{G} \times \Omega^{1}(M): \delta \alpha=0\right\}
$$

and

$$
E:=\operatorname{ker}\left(\left.* d\right|_{E_{0}}\right)^{\perp} .
$$

Note $E$ is a bundle over $\mathcal{G}$ and the operator $* d$ is a fibrewise map. From the Hodge theorem we know $E=\mathcal{G} \times \delta d \Omega^{1}$ and that $* d: E \rightarrow E$ is a bundle isomorphism. Now let $S:=\left\{(g, \alpha) \in E:\|\alpha\|_{2}=1\right\}$ and consider

$$
\phi: S \times \mathbb{R} \rightarrow E \quad ; \quad \phi(g, \alpha, \lambda):=(g, * d \alpha-\lambda \alpha),
$$

so that the inverse image of the zero section gives the curl eigenforms. This is (fibrewise) an index zero Fredholm operator to which the transversality theory detailed in [24] applies. (Though the details are not important, as our operator is elliptic, we will use an appropriate Sobolev completion on the fibers of $E$ and Hölder norms on $\mathcal{G}$.)

Lemma 2.2. For each $r \geq 1$, there exists a residual set in the space of $C^{r}$ metrics on a closed $M^{3}$ such that the eigenspaces of the curl operator (with non-zero eigenvalue) are 1-dimensional and vary smoothly with the metric.

Proof: We will show that the zero-section 0 of $E$ is a regular value of $\phi$. In this case, following [24], $Q:=\phi^{-1}(0)$ is a manifold that fibers over $\mathcal{G}$ with 
projection $\pi$. A $G_{\delta}$-dense set of metrics will be regular values of $\pi$ and, for these values, $Q_{g}=\pi^{-1}(g)=\phi_{g}^{-1}(0)$ is a 0-dimensional manifold (here $\phi_{g}=$ $\left.\left.\phi\right|_{\pi^{-1}(g)}\right)$. For each point $(\alpha, \lambda)$ in $Q_{g}$ we have $* d \alpha=\lambda \alpha$. This $\lambda$ is a simple eigenvalue of curl since 0 is a regular value of $\phi$ (cf. [24, Lemma 2.3]). The eigendecompositions vary smoothly since $Q$ is a manifold. The smoothness condition is required for the application of the Sard-Smale theorem: see [24. p. 1061].

In order to check that 0 is a regular value of $\phi$, we fix $(g, \alpha, \lambda) \in Q$ and consider $\gamma$ orthogonal to the image of $D \phi$. Thus for any $(h, \beta, s) \in T_{(g, \alpha, \lambda)}(S \times$ $\mathbb{R})$ we have

$$
\left\langle * d \beta-\lambda \beta+s \alpha+\left[D_{\mathcal{G}} \phi_{(g, \alpha, \lambda)}\right](h), \gamma\right\rangle=0,
$$

where differentiation along the direction of $\mathcal{G}$ is denoted $\left[D_{\mathcal{G}} \phi_{(g, \alpha, \lambda)}\right]$ (this is denoted $D_{2}$ in [24]). Thus $\gamma$ is orthogonal to $\alpha$ (and so can be thought of as an element of $T_{\alpha} S$ ). Moreover, $\langle\beta, * d \gamma-\lambda \gamma\rangle=0$ for all $\beta \in T_{\alpha} S$. Thus taking $\beta=* d \gamma-\lambda \gamma$ we see that $* d \gamma-\lambda \gamma=0$. (Note $\langle * d \gamma-\lambda \gamma, \alpha\rangle=\langle * d \gamma, \alpha\rangle=$ $\langle\gamma, * d \alpha\rangle=\lambda\langle\gamma, \alpha\rangle=0$ so $* d \gamma-\lambda \gamma \in T_{\alpha} S$.) Hence $\gamma$ is an eigenform for $* d$. Using $\left\langle\left[D_{\mathcal{G}} \phi_{(g, \alpha, \lambda)}\right](h), \gamma\right\rangle=0$ one may easily show that $\gamma$ must vanish in the neighborhood of some point, then unique continuation implies $\gamma=0$. (Alternately one could show $\gamma$ vanishes away from the zeros of $\alpha$ and then use continuity of $\gamma$.)

For such a metric, then, one can unambiguously designate the $i^{t h}$ eigenfield of curl, for $i \in \mathbb{N}$.

Corollary 2.3. Given any continuous $\beta \in E_{\alpha}$ there is an $h$ such that $(h, \beta, 0) \in$ $T_{(g, \alpha, \lambda)} Q$.

Proof: Choose any tangent vector $(h, \beta, 0) \in T_{(g, \alpha, \lambda)} Q$. Being constrained to the tangent space implies that

$$
* d \beta-\lambda \beta+\left[D_{\mathcal{G}} \phi_{(g, \alpha, \lambda)}\right](h)=0 .
$$

From Lemma 2.2 we know that $D_{\mathcal{G}} \phi$ is onto continuous 1-forms (in $E$ ). Therefore, given any $\beta$, we can choose a tangent perturbation $h$ to the space of metrics such that $\left[D_{\mathcal{G}} \phi\right]\left(h_{i}\right)=\lambda \beta_{i}-* d \beta_{i}$, thus solving Equation (2.8).

Lemma 2.4. There is a $G_{\delta}$ dense subset of $C^{r}$ metrics in $\mathcal{G}(r \geq 2)$ for which all curl eigenfields with non-zero eigenvalues have all fixed points nondegenerate.

Proof: Following [24], consider

$$
\psi: Q \times M \rightarrow T^{*} M \quad ; \quad \psi(g, \alpha, \lambda, x):=\alpha(x) .
$$

As in the proof of Lemma 2.2. it suffices to show that the zero-section 0 is a regular value of $\psi$. The smoothness condition is required for the application of the second transversailty theorem of [24, p. 1061] (cf. proof of Theorem 2, p. 1067). Consider an arbitrary point $(g, \alpha, \lambda) \in Q$ and denote 
by $D_{Q} \psi$ the derivative of $\psi$ at this point along $Q$. For $(g, \alpha, \lambda, x) \in Q \times M$, it is clearly seen that $\left[D_{Q} \psi\right](h, \beta, 0,0)=\beta(x)$. Corollary [2.3 then implies that the derivative $D_{Q} \psi$ is onto.

Lemma 2.5. For each $i \in \mathbb{Z}-\{0\}$ and each positive integer $T$, there exists an open dense set of metrics in $\mathcal{G}$ so that, if the $i^{\text {th }}$ eigenfield of curl has no fixed points, then all of the periodic orbits of period less than $T$ are nondegenerate.

Proof: The set of generic (in the sense of Lemma 2.2) metrics in $\mathcal{G}$ which have no fixed points for the $i^{t h}$ eigenfield is open, so work in this set, denoted $\tilde{\mathcal{G}}^{i}$. Choose any metric $g \in \tilde{\mathcal{G}}^{i}$ and let $\tilde{U}_{g}^{i}$ denote a sufficiently small neighborhood of $g$ in $\tilde{\mathcal{G}}^{i}$. Let $u$ denote the $i^{\text {th }}$ curl eigenfield of $g$, and $O_{g}$ the slice of $Q$ through $(g, u, \lambda)$ lying above $\tilde{U}_{g}^{i}$. All Beltrami fields near $u$ are in $O_{g}$. Since there are no fixed points of $u$, the $g$-dual 1-form $\alpha:=\iota_{u} g$ is a contact form whose Reeb field is a rescaling of $u$ by $1 /\|u\|^{2}$. Note that rescalings are irrelevant since nondegeneracy is a topological property of the flowlines of a vector field. The map $(g, u, \lambda) \mapsto\left(\alpha=\iota_{u} g,\|u\|^{2}, J\right)$ is a continuous invertible map (where $J$ is the almost complex structure on the contact structure discussed above) from $O_{g}$ to an open set $U$. Projecting $U$ to the first factor we obtain an open set $U_{\alpha}$ of contact 1-forms near $\alpha$.

We show there is a dense open set in $U_{\alpha}$ containing 1-forms with nondegenerate Reeb vector fields. Then the inverse image of this set will be open and dense in $U$, which leads to an open dense subset of $\tilde{U}_{g}^{i}$ as desired.

Let $\alpha^{\prime}$ be a contact 1-form in $U_{\alpha}$. Gray's theorem (see, e.g., [1. p. 169-171]) says that the perturbed contact structure $\alpha^{\prime}$ can be deformed through a contact isotopy to the contact structure for $\alpha$. Thus, $\alpha^{\prime}$ can be deformed to a 1form which is a near-identity rescaling of $\alpha$. From the proof of Gray's Theorem (using the Moser method in particular), this isotopy is smooth with respect to $\alpha^{\prime}$ - the entire neighborhood of 1 -forms near $\alpha$ can be contactisotoped to near-identity rescalings of $\alpha$.

Fixing the family of contact isotopies, there is an induced continuous map $\Phi$ from $U_{\alpha}$ to $C^{k}(M)$ which returns this well-defined scaling function for the $i^{\text {th }}$ eigenfield of the domain. From results of C. Robinson [22. Thm. 1.B.iv] and Hofer et al. [19, Prop. 6.1], it follows that there is an open dense set of near-identity rescaling functions $f$ such that the Reeb field for $f \alpha$ has all periodic orbits of period $<T$ nondegenerate.

It remains to show that the $\Phi$-inverse image of this open dense set is an open dense subset of $U_{\alpha}$. Since $\Phi$ is continuous, the inverse image is open. One may easily show denseness by observing that if $\Phi\left(\alpha^{\prime}\right) \alpha=\phi^{*} \alpha^{\prime}$ does not have the desired property then there are functions $f_{j}$ arbitrarily close 
to $f=\Phi\left(\alpha^{\prime}\right)$ such that $f_{j} \alpha$ does have the desired property and

$$
\left(\phi^{-1}\right)^{*}\left(f_{j} \alpha\right)=\frac{f_{j} \circ \phi^{-1}}{f \circ \phi^{-1}} \alpha^{\prime}
$$

approximates $\alpha^{\prime}$.

Proof of Theorem 2.1. Take the intersection of the residual sets of metrics from Lemma 2.2. Lemma 2.4 and Lemma 2.5 over all $i$ and $T \in \mathbb{N}$. This intersection has the desired properties.

\section{CONTACT HOMOLOGY}

One of the central problems in the topology of contact structures is the classification problem: given contact structures $\xi$ and $\xi^{\prime}$ on $M$, is there a isotopy of $M$ which takes $\xi$ to $\xi^{\prime}$ ? This problem was greatly clarified by dividing the set of contact structures into two mutually exclusive types: tight and overtwisted structures (for definitions, see [1, p. 192]). A theorem of Eliashberg [6] states that the overtwisted structures are classified by the homotopy type of the plane field, and thus are easily distinguished. This is decidedly not the case for the tight structures, whose classification is a subtle and challenging problem.

To this end, Eliashberg, Givental, and Hofer have announced a powerful new homology theory for contact structures which uses periodic orbits of an associated nondegenerate Reeb field as the chains, a (shifted) ConleyZehnder index as the grading, and pseudoholomorphic curves in the symplectization of the contact manifold as the mechanism for a boundary operator [7|. Very recently, the foundations of this contact homology have been given rigorous proofs in the thesis of Bourgeois [5].

3.1. Introduction to contact homology. Contact homology is an invariant that counts periodic orbits in a Reeb field for a contact structure $\xi$. Specifically, if one fixes a contact structure $\xi$ on $M^{3}$ then one defines the contact homology as follows. Choose a contact 1-form $\alpha$ for $\xi$ and let $X$ be the corresponding Reeb vector field. For a generic choice of $\alpha$, all periodic orbits of $X$ are non-degenerate [19]. Let $\mathcal{C}$ be the set of periodic orbits for the flow of $X$. To each element $c \in \mathcal{C}$, a grading, $|c|$, can be assigned using a shifted Conley-Zehnder index - an integer which is approximately equal to the number of half-twists the linearized flow performs along one cycle. We do not give a precise definition as the only feature of the grading of concern here is:

Lemma 3.1. Any nondegenerate orbit $c$ with $|c|$ odd is hyperbolic.

For a rigorous definition and an explanation of this fact see, e.g., [18]. 
One now defines the graded algebra $\mathcal{A}$ as the free super-commutative unital algebra over $\mathbb{Z}_{2}$ with generating set $\mathcal{C}$. This algebra $\mathcal{A}$ will be the chain "groups" for contact homology. Before defining the boundary map on $\mathcal{A}$ we note one can refine the chains as follows. If $A$ is an element in the first homology of $M$ then one lets $\mathcal{C}_{A}=\left\{c \in \mathcal{C} \mid[c]=A \in H_{1}(M)\right\}$ and defines $\mathcal{A}_{A}$ to be the sub-algebra of $\mathcal{A}$ generated by $\mathcal{C}_{A}$.

The boundary operator is defined in terms of holomorphic curves in the symplectization of $(M, \xi)$. The symplectization of $(M, \xi)$ is the four-manifold $W=M \times \mathbb{R}$ with symplectic form $\omega:=d\left(e^{t} \alpha\right)$, where $t$ denotes the $\mathbb{R}$ coordinate. One now equips $W$ with an almost complex structure $J: T W \rightarrow$ $T W$ by observing that $T_{(x, t)} W=\xi_{x} \oplus \mathbb{R}_{\langle X\rangle} \oplus \mathbb{R}_{\left\langle\frac{\partial}{\partial t}\right\rangle}$, and by defining $J$ on $\xi$ to be any complex structure on $\xi$ compatible with $\left.d \alpha\right|_{\xi}$ and to send $X$ to $\frac{\partial}{\partial t}$. This choice of $J$ entwines the dynamics of $X$ with the $t$-direction.

One next studies holomorphic curves in $W$; that is, maps $\varphi: \Sigma \rightarrow W$ from a Riemann surface $(\Sigma, j)$ to $W$ such that $d \varphi \circ j=J \circ d \varphi$. It is a fact that there are no compact Riemann surfaces in $W$; one must introduce punctures [18]. If $\Sigma$ is a punctured Riemann surface, the energy of $\Sigma$ is defined to be $\int_{\Sigma} \varphi^{*}(d \alpha)$. If $\varphi=(w, h): \Sigma \rightarrow M \times \mathbb{R}$ has finite energy then some punctures may be removable. Those that are not removable are guaranteed to possess a neighborhood parametrized by $\left\{(\theta, \tau): \theta \in S^{1}\right.$ and $\left.\tau \in[0, \infty)\right\}$ such that $\lim _{\tau \rightarrow \infty} h$ approaches $\pm \infty$ and $\lim _{\tau \rightarrow \infty} w(\theta, \tau)$ approaches a parametrization of a periodic orbit $\gamma$ for $X$. The intuition behind this is that if a surface has finite energy, then in the limit as $t \rightarrow \pm \infty$, the surface must be orthogonal to the contact planes, and thus tangent to the $\left(X, \frac{\partial}{\partial t}\right)$ planes. One calls a puncture positive (negative) if $h$ approaches $\infty(-\infty)$ as $\tau \rightarrow \infty$. For more information on finite energy holomorphic curves and their asymptotics see [4].

Given periodic orbits $a, b_{1}, \ldots, b_{k} \in \mathcal{C}$, let $\mathcal{M}_{b_{1} \ldots b_{k}}^{a}$ denote the set of finite energy holomorphic curves in $W$ with one positive puncture asymptotic to $a$ and negative punctures asymptotic to $b_{1}, \ldots, b_{k}$, modulo holomorphic reparametrization. Note: since $J$ is $\mathbb{R}$-invariant, there is an $\mathbb{R}$-action on $\mathcal{M}$. One now defines

$$
\partial a=\sum\left(\# \mathcal{M}_{b_{1} \ldots b_{k}}^{a} / \mathbb{R}\right) b_{1} \ldots b_{k},
$$

where the sum is taken over all $b_{1}, \ldots b_{k}$ such that the dimension of $\mathcal{M}_{b_{1} \ldots b_{k}}^{a}$ is 1 .

Proposition 3.2. [5, 7] The differential $\partial$ lowers grading by 1. For a generic contact 1-form (and almost complex structure) $\partial^{2}=0$ and the homology of $(\mathcal{A}, \partial)$ is independent of the contact form chosen for $\xi$ (and the almost complex structure). 
The homology of $(\mathcal{A}, \partial)$ is called the contact homology of $(M, \xi)$ and is denoted $C H(M, \xi)$. It is also useful to consider the contractible contact homology $\mathrm{CH}_{0}(M, \xi)$ whose chain groups are $\mathcal{A}_{[0]}$, the algebra generated by contractible periodic orbits. The contractible contact homology is also well defined for generic contact forms.

It is in general difficult to find all holomorphic curves in $W$ and hence to compute the contact homology. It is frequently easier to compute the cylindrical contact homology. Here one uses $\mathcal{A}_{A}$ as the chain groups and when one defines the boundary map one uses only holomorphic cylinders $\Sigma=S^{1} \times \mathbb{R}$.

Proposition 3.3. [5, 25] The cylindrical contact homology $\mathrm{CCH}_{A}(M, \alpha)$ is welldefined and independent of the contact form used so long as there are no contractible periodic orbits with grading $-1,0$ or 1 .

3.2. The 3-torus. Proving the hydrodynamic instability theorem requires knowing the existence of a periodic orbit of saddle-type for all non-degenerate Reeb fields on $T^{3}$. A deep theorem of Hofer [18] guarantees that any Reeb field for an overtwisted contact structure possesses a closed orbit of grading +1 ; thus, we need merely cover the case of the tight contact structures. For $T^{3}$, these are fortunately classified [16, 20]: there is an infinite family of isomorphism classes represented by

$$
\xi_{k}:=\operatorname{ker}(\sin (k z) d x+\cos (k z) d y),
$$

for $k \in \mathbb{Z}-\{0\}$. The following contact homology argument is the crucial step in the instability proof:

Lemma 3.4. For a nondegenerate Reeb field associated to any tight contact structure on $T^{3}$, there is always hyperbolic periodic orbit.

Proof. We begin with an explicit cylindrical homology computation which controls the grading of orbits. For any generic rescaling of the forms in Eqn. (3.2) for which cylindrical contact homology is well-defined, Bourgeois [5] has shown that $C C H_{A}\left(T^{3}, \xi_{k}\right)$ is nontrivial in grading -1, where $A$ is, say, the homology class given by $\{(x, y, z): y=0, z=0\}$. Thus there must be hyperbolic periodic orbits whenever the cylindrical contact homology is well defined. If the cylindrical contact homology is not welldefined then there must be contractible periodic orbits with grading either $-1,0$ or 1 via Proposition 3.3 If the gradings are 1 or -1 then there exists a contractible hyperbolic periodic orbit.

In the case that the grading is 0 , we turn to the full contact homology. by using the Bott-Morse perturbation technique of [5], one shows that there are no contractible periodic orbits for nondegenerate contact forms close to those of Eqn. (3.2). Hence, the contractible contact homology of $\left(T^{3}, \xi_{k}\right)$ vanishes. Since, in the case considered, we must have a contractible periodic orbit with grading zero, the chain complex for $\mathrm{CH}_{0}\left(T^{3}, \xi_{k}\right)$ possesses 
an element $a$ with $|a|=0$. Therefore, there must exist a nontrivial chain with odd grading which prevents a nonzero cycle in the contact homology. This implied chain is the desired hyperbolic orbit.

\subsection{Proof of main theorem.}

Theorem 3.5. For generic choice of $C^{r}$ metric $(2 \leq r<\infty)$, all of the curleigenfields on a three-torus $T^{3}$ (with nonzero eigenvalue) are hydrodynamically unstable.

Proof: First, use Theorem 2.1 to reduce everything to either nondegenerate fixed points or periodic orbits. Given such a field $u$, if it possesses a fixed point, then it is immediately of saddle type due to volume conservation and satisfies the Instability Criterion. If the field is free of fixed points, then it is (after a suitable rescaling which preserves the topology of the flowlines) a Reeb field for the contact form $\alpha:=\iota_{u} g$. If the contact structure $\xi=\operatorname{ker} \alpha$ is overtwisted, Hofer's theorem [18] implies the existence of a periodic orbit with grading +1 . The nondegeneracy implies that the orbit is of saddle type and hence forces hydrodynamic instability. In the final case where $\xi$ is tight, the contact homology computation of Lemma 3.4 implies instability.

\section{CONCLUSIONS AND QUESTIONS}

With the exception of the contact homology computation for the class of tight contact structures on $T^{3}$, the methods used to prove generic instability are applicable to any closed three-manifold, as well as to compact three-manifolds with invariant boundaries (e.g., the solid torus). On a few sufficiently simple three-manifolds (spheres, lens spaces), it is possible to have nondegenerate curl eigenfields which do not possess hyperbolic periodic orbits. It remains unclear whether these fields are hydrodynamically unstable.

Besides the curl eigenfields, the only other steady solutions to the Euler equations in 3-d are integrable: the flow domain is filled almost everywhere with invariant 2-tori [2]. The only viable candidates for integrable fields which are not hydrodynamically unstable are those which possess a great deal of symmetry, e.g., the Hopf flow (unit flow along the fibers of the Hopf fibration on the unit 3-sphere in Euclidean $\mathbb{R}^{4}$ ).

Question 4.1. Is the Hopf flow on the round $S^{3}$ linearly unstable?

\section{REFERENCES}

[1] B. Aebischer, M. Borer, M. Kählin, Ch. Leuenberger, and H. M. Riemann, Symplectic Geometry. Birkhäuser, 1994.

[2] V. I. Arnold, Sur la géométrie differentielle des groupes de Lie de dimension infinie et ses applications à l'hydronamique des fluides parfaits. Ann. Inst. Fourier (1966) 16, 316-361. 
[3] V. I. Arnold and B. Khesin, Topological Methods in Hydrodynamics. Springer-Verlag, 1998.

[4] M. Audin, J. Lafontaine, eds. Holomorphic Curves in Symplectic Geometry. Birkhäuser, 1994.

[5] F. Bourgeois, A Morse-Bott approach to contact homology, PhD Thesis, Stanford 2002.

[6] Y. Eliashberg, Classification of overtwisted contact structures on 3-manifolds. Invent. Math. 98 (1989), 623-637.

[7] Y. Eliashberg, A. Givental and H. Hofer, Introduction to symplectic field theory, GAFA 2000 (Tel Aviv, 1999), Geom. Funct. Anal. (2000), Special Volume, Part II, 560-673.

[8] J. Etnyre and R. Ghrist, Contact topology and hydrodynamics I. Beltrami fields and the Seifert conjecture. Nonlinearity 13 (2000), no. 2, 441-458.

[9] J. Etnyre and R. Ghrist, Contact topology and hydrodynamics II. Solid tori. Ergodic Theory Dynam. Systems 22 (2002), no. 3, 819-833.

[10] J. Etnyre and R. Ghrist, Contact topology and hydrodynamics III. Knotted flowlines. Trans. Amer. Math. Soc. 352 (2000), 5781-5794.

[11] C. Foias and R. Temam, Structure of the set of stationary solutions of the Navier-Stokes equations, Comm. Pure \& Appl. Math. XXX (1977) 149-164.

[12] S. Friedlander, Lectures on stability and instability of an ideal fluid, in Hyperbolic equations and frequency interactions (Park City, UT, 1995), 227-304, IAS/Park City Math. Ser., 5, Amer. Math. Soc., Providence, RI, 1999.

[13] S. Friedlander and M. Vishik, Instability criteria for steady flows of a perfect fluid. Chaos 2 (1992), no. 3, 455-460.

[14] S. Friedlander and V. Yudovich, Instabilities in fluid motion. Notices Amer. Math. Soc. 46 (1999), no. 11, 1358-1367.

[15] R. Ghrist and R. Komendarczyk, Topological features of inviscid flows, in Geometry and Topology of Fluids, NATO ASI Series II vol. 47, Kluwer Press, 183-202.

[16] E. Giroux, Une infinité de structures de contact tendues sur une infinité de variétés. Invent. Math. 135 (1999), no. 3, 789-802.

[17] D. Henry, Generic properties of equilibrium solutions by perturbation of the boundary. Dynamics of infinite-dimensional systems (Lisbon, 1986), 129-139, NATO Adv. Sci. Inst. Ser. F Comput. Systems Sci., 37, Springer, Berlin, 1987.

[18] H. Hofer, Pseudoholomorphic curves in symplectizations with applications to the Weinstein conjecture in dimension three. Invent. Math. 114 (1993), no. 3, 515-563.

[19] H. Hofer, K. Wysocki, and E. Zehnder, The dynamics on three-dimensional strictly convex energy surfaces. Ann. of Math. (2) 148 (1998), no. 1, 197-289.

[20] Y. Kanda, The classification of tight contact structures on the 3-torus. Comm. Anal. Geom. 5 (1997), no. 3, 413-438.

[21] A. Lifshitz and E. Hameiri, Localized instabilities of vortex rings with swirl. Comm. Pure Appl. Math. 46 (1993), no. 10, 1379-1408.

[22] C. Robinson, Generic properties of conservative systems. Amer. J. Math. 92 (1970), 562-603.

[23] J. Saut and R. Temam, Generic properties of Navier-Stokes equations: genericity with respect to the boundary values, Indiana Univ. Math. J. 29 (1980), no. 3, 427-446.

[24] K. Uhlenbeck, Generic properties of eigenfunctions, Amer. J. Math., 98 (1976), no. 4, 10591078.

[25] I. Ustilovsky, Infinitely many contact structures on $S^{4 m+1}$, Internat. Math. Res. Notices, 14 (1999), 781-791.

Department of Mathematics, University of Pennsylvania, Philadelphia PA, 19104-6395

DEPARTMENT OF MATHEMATICS, UNIVERSITY OF ILLINOIS, URBANA IL, 61801 